\title{
Analyzing Event-Related Brain Dynamics in Continuous Compensatory Tracking Tasks
}

\author{
Ruey-Song Huang ${ }^{1,2}$, Tzyy-Ping Jung ${ }^{2}$ and Scott Makeig ${ }^{2}$ \\ ${ }^{1}$ Department of Cognitive Science, University of California, San Diego, USA \\ ${ }^{2}$ Swartz Center for Computational Neuroscience, Institute for Neural Computation, University of California, San Diego, USA
}

\begin{abstract}
The dynamics of electroencephalographic (EEG) activity in continuous compensatory tracking tasks were analyzed by independent component analysis (ICA) and time-frequency techniques. In one-hour sessions, 72-channel EEG was recorded while a healthy volunteer attempted to use a trackball to keep a drifting disc in a bulls-eye in the center of screen. Disc trajectory was converted into a moving measure of disc error. Local minima (perigees) indicated moments when the disc started to drift away from the center. Subject performance was indexed by root mean square disc error in a 20s epoch centered on each perigee, high error generally indicating drowsiness. Maximally independent EEG processes and their equivalent dipole source locations were obtained using the EEGLAB toolbox (http://sccn.ucsd.edu/eeglab). Component activations were epoched in $5 \mathrm{~s}$ time intervals time locked to perigees. Following disk perigees during (drowsy) periods of high disk error, significant spectral changes were observed. One of the 70 independent components was located in or near primary visual cortex. During periods of poor (drowsy) performance, it had increased mean tonic alpha/theta activity, with a further phasic alpha/theta increase following perigees $[1,2]$. At the same time, low alpha activity of a second component located in or near cingulate gyrus increased, and 10-30 Hz EEG activity of a third component in the left somatomotor cortex increased briefly. The alpha activity of the somatomotor component persisted through the following distance maximum. These spatiotemporal phenomena were consistently observed across three sessions within subjects. Thus, event-related EEG brain dynamics can be detected and modeled in a continuous behavioral task without impulsive event onsets.
\end{abstract}

Index Terms -- EEG, ICA, ERSP, continuous task, performance, brain dynamics, theta, alpha, beta.

\section{INTRODUCTION}

Sensory event-related potentials (ERP) index mean electroencephalographic (EEG) activities following onsets of visual or auditory stimuli. In many ERP paradigms, participants respond to stimuli with discrete button presses. ERPs are usually obtained by time-domain average of EEG epochs precisely phase-locked to stimulus or to response onsets. In real-life, many tasks require more or less continuous efforts to maintain appropriate behavior, instead of discrete (button) choice responses. During the course of truly continuous performance paradigm, on the other hand (e.g., in driving simulations), participants can receive continuous visual and/or auditory stimuli as well as continuous performance feedback. The ERP averaging technique is limited to tasks with discrete stimulus events and may require a silent baseline period preceding stimulus onsets, making it of little use for assessing brain dynamics in continuous performance tasks.

In this study, we applied event-related spectral perturbation (ERSP) methods to study event-related brain dynamics in a continuous compensatory tracking task (CTT) during which participants attempted to use a trackball to keep a randomly drifting disc in a bulls-eye on the center of screen [3]. Independent component analysis (ICA) was applied to continuous EEG data collected in each of the 1-hour CTT sessions. Maximally independent EEG processes and their dipole source locations were obtained by the EEGLAB toolbox (http://sccn.ucsd.edu/eeglab) [4]. During high-error periods, three clusters of independent components exhibited significant spectral perturbations following disc trajectory 'perigees' (moments when the disc began to escape from the center). Within subjects, the component activations and scalp topographies of these clusters were stable across sessions. This study also demonstrates that brain dynamics linked to changes in human performance can be assessed on the sub-second time scale in a continuous performance task.

\section{MATERIALS AND MeTHODS}

\section{A. Participants and Tasks}

Six right-handed adults (3 males, 3 females, mean age $=$ $27.8, \mathrm{SD}=6.0$ ) with normal or corrected to normal vision volunteered to participate in this experiment. Informed consent was obtained from all participants. Participants arrived after lunch and sat on a cozy chair in the EEG booth in which lighting was dim. Each subject participated a three one-hour sessions of continuous visuospatial compensatory tracking tasks in which they attempted to use a trackball to keep a drifting ('wind-blown') disc in a bulls-eye as near as possible to the center of screen (Fig. 1). Participants were instructed to continue to perform the task as best as they could even when they began to feel drowsy. No intervention was made when participants occasionally fell asleep and stopped responding; participants resumed task performance themselves after non-responding periods. The coordinates of the drifting disc and trackball activities were recorded about 14 times per second while a synchronous pulse marker train was sent to the EEG recording system. 
Due to space constraint, data from one participant are reported here.

\section{B. EEG Recordings}

EEG activities were recorded from 69 scalp electrodes. The reference was the right mastoid. Eye movements and blinks were recorded via two EOG electrodes placed below the right eye and at the left outer canthus, respectively. EEG and EOG activities were sampled at $250 \mathrm{~Hz}$ with an analog pass band of 0.01-100 Hz. Data were digitally filtered (1-45 $\mathrm{Hz}$ pass band).

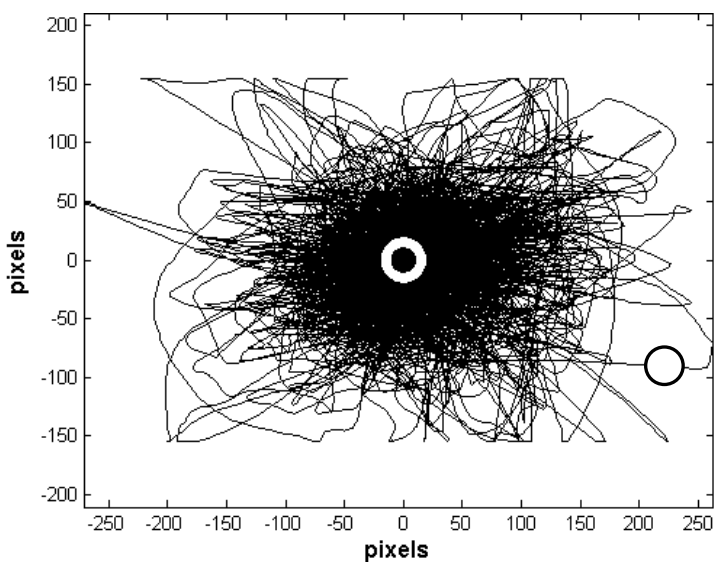

Fig. 1. Disc trajectory from a one-hour session. The white ring represents the bulls-eye. The black circle represents the drifting disc whose movements were partially controlled by the subject through trackball motions

\section{Analysis of Tracking Performance}

Fig. 1 demonstrates an accumulated disc trajectory from a 1-hour CTT session (SY01). Time series of disc coordinates, $\mathrm{x}(\mathrm{t})$ and $\mathrm{y}(\mathrm{t})$, were converted into a time series of disc error $\mathrm{d}(\mathrm{t})$, defined as the radial distance between the disc and the center of the screen. Tracking performance was obtained by taking root mean square (RMS) of $d(t)$ in a designated time window. RMS of disc errors in a short moving average window (e.g., 4 seconds) reflects the participant's local tracking performance, whereas averaging performance in a longer window (e.g., 20 seconds) reflects more global changes in tracking performance. Fig. 2(a) illustrates 2-D disc trajectory in a 4-s window (green and red curves) and in a 20-s window (cyan curve). Fig. 2(b) illustrates the time series of disc error $d(t)$ in the 4-s window. The green and red curves represent disc trajectory from $2 \mathrm{~s}$ before to $2 \mathrm{~s}$ after the local minimum or perigee, the moment when the disc starts to drift away from the bulls-eye. The perigee was used as a time-locking event, and data epochs time-locked to perigees as single trials in which participants had to attempt to use the trackball to return the disc back toward the central ring. Participants' motor responses were recorded by two time series, the trackball velocity, $\mathrm{V}_{\mathrm{x}}(\mathrm{t})$ and $\mathrm{V}_{\mathrm{y}}(\mathrm{t})$. The blue curve in Fig. 2(b) represents the magnitude of trackball velocity, and each peak representing a trackball movement. The first velocity peak following a perigee was defined as the subject's initial response.

In session SY01, 1814 perigees were extracted from time series $d(t)$. RMS of disc errors in 4-s (local) and 20-s (global) epochs centered at the perigee of each trial were evaluated. Fig. 3(a) demonstrates local and global RMS disc error in chronological order, including several prolonged fluctuations in tracking performance during the hour-long session. Fig. 3(b) demonstrates the extracted trials sorted by RMS disc error. Here, values near 0 reflect optimal compensatory tracking.

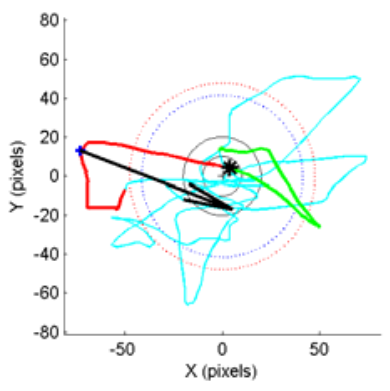

(a)

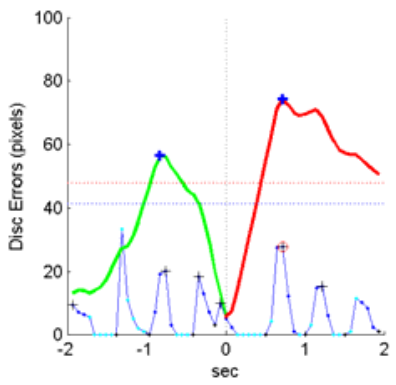

(b)
Fig 2. (a) Disc trajectory. Two concentric black rings represent the bull's-eyes at the center of the screen. Participants were asked to keep the disc in the inner ring. The cyan curve represents the disc trajectory in a 20-s epoch, and the radius of the blue dashed ring is the RMS disc error in the 20-s epoch. The black asterisk in the inner ring represents the local minimum (perigee). The green (red) curve represents 2-s disc trajectory entering (leaving) the local minimum. The radius of the red dashed ring represents the RMS of disc error in the 4-s epoch. The black arrow represents the direction and amplitude of instantaneous trackball velocity. (b) Time series of disc error, $\mathrm{d}(\mathrm{t})$, and magnitude of trackball motion during the 4-s epoch. The green and red curves represent the disc error before and after the perigee, respectively. The blue curve represents trackball velocity. A blue (cyan) dot indicates correct (incorrect) direction of response. Each black cross represents a trackball movement. The black cross enclosed by a small red circle represents the initial trackball response after the local minimum. The red and blue dashed lines represent RMS disc error in the 4-s and 20 -s epochs, respectively.
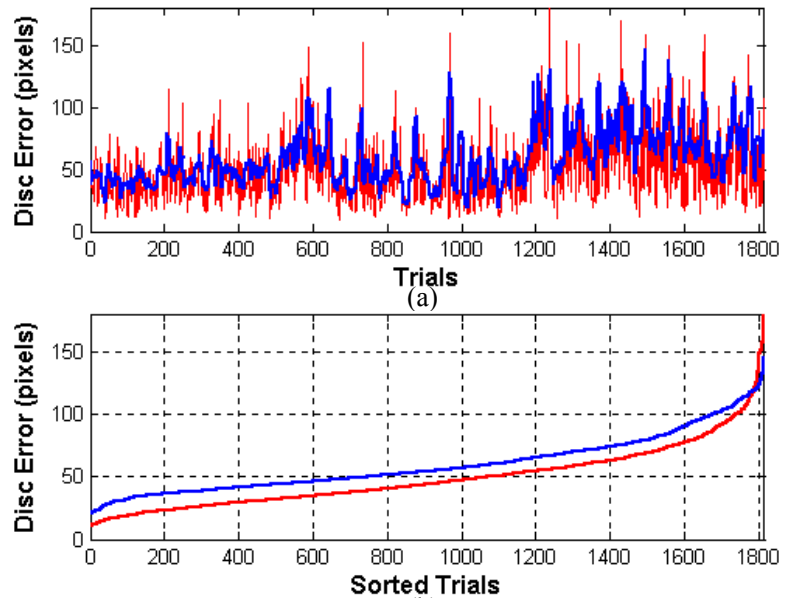

(b)

Fig. 3. (a) Time series of local (4-s, red) and global (20-s, blue) RMS disc error. (b) Sorted RMS disc error curves.

\section{Artifact Rejection}

This experiment required continuous effort and frequent hand and finger movements, sometimes accompanied by head or neck muscle twitch artifacts in the EEG data. In addition, a few times participants felt drowsy and yawned during the sessions. These events caused severe artifacts 
across all channels in some epochs, which were rejected from the continuous EEG data using the EEGLAB toolbox [4]. Bad channel records produced by poor skin contacts were also rejected. Other sources of artifacts (blinks, eye movements, and head-muscle artifacts), were separated from other EEG processes using ICA as described below.

\section{E. ICA and Independent Component Selection}

Maximally independent EEG processes were obtained using the runica algorithm of the EEGLAB toolbox. ICA was trained separately for each session of each subject. Each ICA training set consisted of 68-71 channels of continuous data, approximately 2000-3500 s in length. Initial learning rate was $10^{-4}$; training was stopped when learning rate fell below $10^{-6}$. Seventy independent components were obtained from session SY01 (3322 s, 70 channels). Some components were identified as accounting for blinks, eye-movements and muscle artifacts [5]. Components of interest were selected based on their characteristic scalp maps, dipole source locations, spectral signatures, and within subject consistency [6]. Three clusters of components with equivalent dipoles in occipital, somatomotor, and frontal midline cortex are studied in this report. Fig. 4 plots a component originating in bilateral occipital cortex plus its power spectrum (see Results) .

\section{F. Trials Selection and Epoch Segmentation}

Each local minimum in $\mathrm{d}(\mathrm{t})$ corresponds to a disk perigee. The average inter-perigee-interval (IPI) of the 1814 trials in session SY01 was about 2s. Three criteria were used for trials selection. First, involuntary subject finger movement resulted in many small and transient dips in $\mathrm{d}(\mathrm{t})$. Therefore, trials that were followed by an IPI of less than $1.2 \mathrm{~s}$ were not selected for further analysis. Second, trials in which the subject made no motor response 200 to $2000 \mathrm{~ms}$ after the perigee were rejected. Third, trails contaminated by artifacts (excluding blinks and eye movements) were rejected. In session SY01, 937 out of 1814 trails were thus selected for time-frequency analysis. Component activations were epoched in 5-s time intervals, 2-s preceding and 3-s following each perigee. In session SY01, 937 5-s epochs (trials) were selected for further analysis.

\section{G. Error-Sorted Component Activity Power}

The traditional ERP approach, using time-domain averages of epochs from two or more task conditions, is not applicable to epochs with no discretely perceived time-locking event, as in this paradigm. The erpimage() function of the EEGLAB toolbox was used to visualize power of component activity in the selected epochs sorted in order of tracking performance, i.e. by local or global RMS disc errors (Fig. 5 in Results).

\section{H. Time Frequency Analysis (ERSP)}

The event-related spectral perturbation (ERSP) transform, newtimef() of EEGLAB was applied to the selected epochs of each component activation. Fig. 6 shows ERSPs of the same occipital component in two conditions (alert and drowsy) plus their statistically significant difference. Selected epochs are shown in Fig. 5 (epochs in the dashed and dotted boxes, respectively). The ERSP images only show significant $(\mathrm{p}<0.01)$ spectral perturbation relative to the (alert) power spectrum baseline (before the perigee). Non-significant time/frequency points are colored in green.

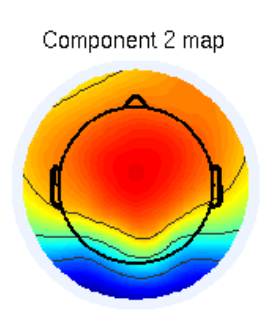

(a)

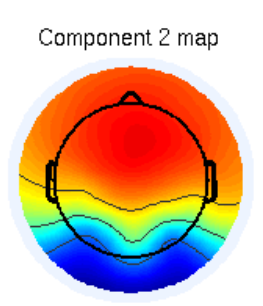

(c)

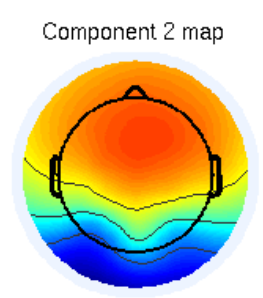

(e)

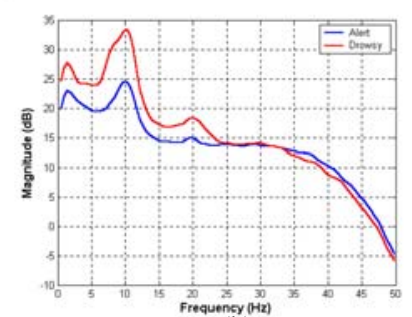

(b)

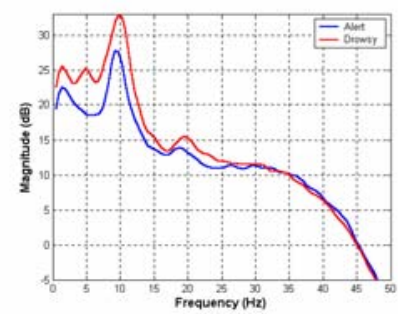

(d)

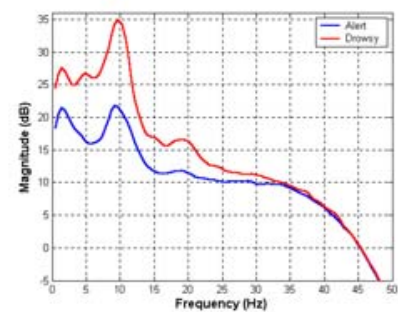

(f)
Fig. 4. Scalp maps (a, c, e) and power spectra (b, d, f) of an occipital components separately derived from sessions SY01, SY02, SY03. The blue/red curves show the average power spectrum in low- and high-error epochs, i.e. in periods of alert and drowsy performance, respectively.

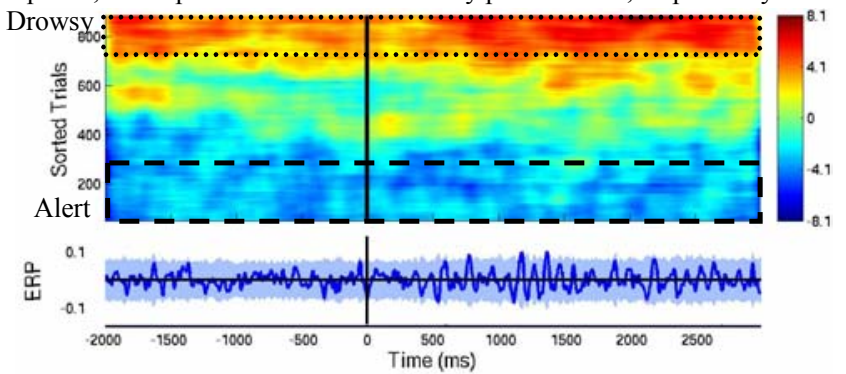

Fig. 5. (Top panel) Alpha (8-12 Hz) power in global RMS error-sorted epochs. The dashed box contains the most alert $30 \%$, and the dotted box the $20 \%$ most drowsy epochs. (Bottom trace) Time-average ERP of all the epochs.

\section{RESULTS}

\section{A. Occipital Components}

Fig. 4 shows an occipital component, whose scalp map and spectrum were consistently recovered from three sessions of the same subject. Alpha (8-12 Hz) activity of this component was stronger in epochs exhibiting poor (drowsy) tracking performance.

Fig. 5 shows the alpha power of the occipital component in epochs sorted by global disc error (alert performance at the bottom). The image shows increased tonic alpha 
activity in periods of poor (drowsy) performance. Typical visual ERP features, e.g. N1 and P1, were not observed in the (n.s., blue band) time-averaged ERP of the same epochs.

Fig. 6 illustrates drowsy and alert condition ERSPs for the same occipital component separated from two sessions of the same subject. The drowsy-alert ERSP difference contains tonic high and low alpha activities, indicating a tonic EEG difference between alertness and drowsiness. In addition, the alert condition ERSP (left) shows phasic alpha and theta increases following the disk perigee (vertical dashed line).

\section{B. Sensorimotor-Related Components}

Fig. 7(a) shows scalp map, drowsy condition ERSP, and drowsy-alert ERSP difference for a somatomotor component from session SY01 (also found in other sessions). Phasic EEG activity between 10 and $30 \mathrm{~Hz}$ increased following perigees, alpha activity persisting even after the local maximum (apogee) was reached.

\section{Midline Component}

Fig. 7(b) shows the scalp map and ERSP difference for another component from session SY01 located in or near the cingulate gyrus (also observed in other sessions). Following perigees, low alpha activity of this component increased.
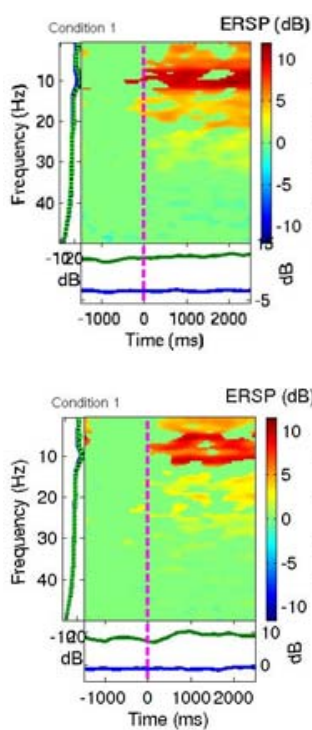

Fig. 6. (a) ERSPs in two conditions of the same occipital component in session SY01. Left: ERSP in Condition 1 (drowsy state; worst $20 \%$ of global RMS error-sorted trials). Middle: ERSP in Condition 2 (alert state, best $30 \%$ of global RMS error-sorted epochs). Right: ERSP differences between Conditions 1 and 2 (drowsy - alert). (b) Similar ERSPs for the equivalent occipital component from session SY02 (Fig. 4(c,d)).

\section{CONCLUSION}

This study demonstrates tonic and phasic event-related brain dynamics accompanying human performance in a realistic setting. The moment when the disc starts to escape from the center of the screen is consider an event embedded in a continuous tracking task. Neither the experimenter nor the participant can predict the precise timing of event onsets. Event onsets can be obtained by analyzing the continuous trajectory of the drifting disc off-line. Maximally independent EEG processes were obtained using the EEGLAB toolbox. Three clusters of components, located in occipital, somatomotor and cingulate cortex, showed significant spectral perturbation when the disc starts to drift away. The time-frequency patterns of these components were consistently observed across three sessions from a subject. Thus, changes of EEG brain dynamics $[6,7]$ can be modeled in a continuous behavioral task.
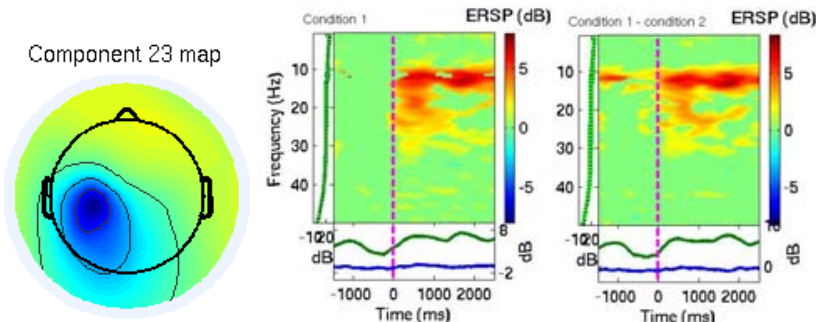

(a)
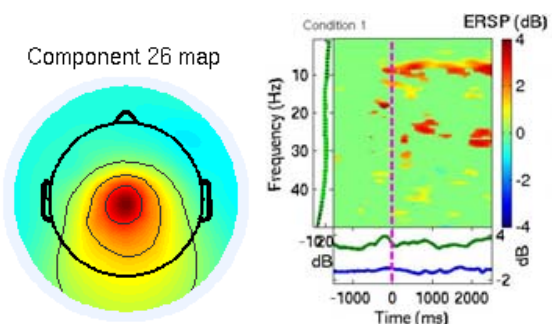

(b)

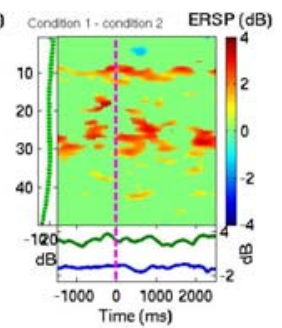

Fig. 7. (a) Left: Scalp map of a somatomotor component from session SY01. Middle: ERSP in Condition 1 (drowsy state; worst $20 \%$ global error trials). Right: ERSP difference (drowsy - alert). (b) Left: Scalp map of a midline component from session SY01. Middle and Right: As in (a).

\section{ACKNOWLEDGEMENT}

Research supported by The Swartz Foundation (Old Field NY), NASA, DENSO Corp., and Office of Naval Research.

\section{REFERENCES}

[1] S. Makeig, and T.-P. Jung, "Tonic, phasic and transient EEG correlates of auditory awareness in drowsiness," Cogn Brain Res, vol. 4, pp. 15-25, 1996.

[2] T.-P. Jung, S. Makeig, M. Stensmo, and T.J. Sejnowski, "Estimating alertness from the EEG power spectrum," IEEE Trans Biomed Eng, vol. 44, no. 1, pp. 60-69, 1997.

[3] S. Makeig. and M. Jolley, "COMPTRACK: A compensatory tracking task for monitoring alertness." http://sccn.ucsd.edu/ scott/.

[4] A. Delorme and S. Makeig, "EEGLAB: an open source toolbox for analysis of single-trial EEG dynamics including independent component analysis," J. Neurosc. Meth., vol. 134, pp. 9-21, 2004.

[5] T.-P. Jung, C. Humphries, T.-W. Lee, M.J. McKeown, V. Iragui, S. Makeig, and T.J. Sejnowski, "Removing electroencephalographic artifacts by blind source separation," Psychophysiology, vol. 37, pp. 163-178, 2000.

[6] T.-P. Jung, S. Makeig, M.J. McKeown, A.J. Bell. T.-W. Lee, and T.J. Sejnowski, "Imaging brain dynamics using independent component analysis," Proc. IEEE, vol. 89, no. 7, pp.1107-1122, 2001.

[7] S. Makeig, S.Debener, J. Onton, and A. Delorme, "Mining event-related brain dynamics." Trends in Cognitive Science, vol. 8, no. 5, pp. 204-210, 2004. 